\title{
CHRONIQUES D'HABITAT ET DYNAMIQUE DE POPULATIONS DE TRUITE.
}

\author{
H. CAPRA (1), S. VALENTIN (1) et P. BREIL (2)
}

(1) Division Biologie des Ecosystèmes Aquatiques, Laboratoire d'Hydroécologie Quantitative, CEMAGREF, 3 bis Quai Chauveau, CP 220, 69336 Lyon Cedex 09, France.

(2) Division Hydrologie-Hydraulique, CEMAGREF, 3 bis Quai Chauveau, CP 220, 69336 Lyon Cedex 09, France.

\section{RÉSUMÉ}

Une nouvelle méthode a permis de quantifier des Durées Continues d'Habitat Limitant (méthode $\mathrm{DCHL}$ ) à partir de chroniques d'habitat potentiel, pour la truite fario (Salmo trutta fario, L.). Cette méthode a été appliquée sur deux cours d'eau naturels et sur une station soumise à éclusée. Elle a été validée sur les deux cours d'eau naturels en ce qui concerne l'habitat limitant pour les jeunes truites de l'année $(0+)$. Elle offre un outil pratique d'analyse des périodes d'habitat limitant en régime naturel. En situation d'éclusées, l'interprétation des résultats est différente et nécessite encore un effort important de suivi biologique.

\section{HABITAT TIME SERIES AND TROUT POPULATION DYNAMICS.}

\section{ABSTRACT}

A new tool was used to quantify Continuous Under Threshold Habitat Duration Curves (CUT curves) from habitat chronicles, for brown trout (Salmo trutta fario, L.). It was tested in two natural streams and in a regulated site with intermittent peak discharge. It was validated in the natural streams regarding the limiting habitat of young brown trout of the year $(0+)$. In the regulated station, results suggested that biological surveys are necessary to identify accurate limiting habitat.

\section{INTRODUCTION}

D'importants progrès ont été réalisés au cours des deux dernières décennies dans la compréhension des relations entre les compartiments morphodynamiques et biologiques des cours d'eau (GORE et al., 1994). Les recherches dans ce domaine ont permis de mettre au point des outils de prédiction de l'habitat physique potentiel pour les poissons, en particulier pour aider à la détermination des débits réservés en aval des barrages. Un des outils les plus utilisés aux Etats-Unis (REISER et al., 1989) est la méthode IFIM (Instream Flow Incremental Methodology, BOVEE, 1982), incluant une simulation de l'habitat physique potentiel en fonction du débit (Physical HABitat SIMulation, MILHOUS et al., 1989). Cette approche a été adaptée en France sous le nom de méthode des microhabitats (SOUCHON et al., 1989) ou, plus récemment EVHA (EValuation de l'HAbitat, GINOT et SOUCHON, 1992). Dans un premier temps, les validations biologiques ont consisté en une comparaison d'un habitat potentiel à un débit donné avec une image des populations de truite en place à un moment particulier (JOWETT, 1992 ; SHULER et NEHRING, 1994). Le caractère statique de la simulation des conditions d'habitat a entraîné des critiques sur la validité de la méthode (ORTH, 1987 ; SCOTT et SHIRVELL, 1987). Les connaissances sur la dynamique des populations soulignent la nécessité d'intégrer la dimension temporelle afin d'analyser les périodes d'habitat limitant selon les stades de développement, en durée et en fréquence (ORTH, 1987). 
Une méthode toute récente permet de lire des chroniques d'habitat, à l'aide d'outils utilisés en hydrologie pour décrire et analyser les variations de débit (OBERLIN et al., 1989). Cette méthode propose une représentation synthétique des Durées Continues d'Habitat Limitant, DCHL (CAPRA et al., 1994). Nous proposons ici d'analyser la variabilité des conditions d'habitat à l'aide de cette méthode, d'une part en situation naturelle, dans des conditions non exceptionnelles et, d'autre part, en situation d'éclusées hydroélectriques. Pour aider à l'interprétation des résultats obtenus en milieu naturel, une première phase de validation biologique en milieu naturel a été réalisée par CAPRA et al. (1994), montrant l'influence des durées continues d'habitat limitant pendant la reproduction sur les effectifs de $0+$ de truite fario (Salmo trutta fario, L.). Une deuxième phase de validation biologique est proposée ici, également en situation naturelle, à partir de l'étude des variations d'habitat potentiel pour la cohorte $0+$ en relation avec les effectifs de $0+$.

\section{MATÉRIELS ET MÉTHODES}

\section{Sites d'étude}

La Semine (Ain) et la Boralde de St Chély (Aveyron) sont deux cours d'eau de référence, sur les tronçons étudiés. Leur régime hydrologique et leur qualité physicochimique sont naturels. Leur peuplement est salmonicole (truite seule sur la Semine et truite accompagnée de chabot sur St Chély). L'alevinage et la pression de pêche sont faibles, n'entraînant pas de modification importante de la dynamique des populations de truite autochtone. Ce sont des cours d'eau à pente élevée associée à une granulométrie grossière et à une végétation rivulaire arborescente abondante. Leurs modules sont égaux et de $1.6 \mathrm{~m}^{3} \cdot \mathrm{s}^{-1}$. Sur chaque rivière, une station d'étude a été choisie représentative de la géomorphologie du tronçon, c'est-à-dire comportant une diversité de conditions d'habitat suffisante pour accueillir tous les stades de développement de la truite (POUILLY et al., 1995).

\section{Evaluation de l'habitat pour la cohorte 0+}

Sur chacune des stations étudiées, une description physique a été réalisée selon le protocole de la méthode des microhabitats pour obtenir une modélisation de l'habitat potentiel en fonction du débit (POUILLY et al., 1995). La caractérisation physique de chaque station consiste en une description topographique et hydraulique sur plusieurs transects, aboutissant à un découpage en petites surfaces élémentaires (ou cellules) à caractéristiques physiques homogènes dans le sens longitudinal et latéral. Les variables physiques mesurées sont la hauteur d'eau $(H)$, la vitesse $(V)$ du courant et le substrat $(S)$. Chaque valeur de $H, V$ et $S$ est transformée en coefficient de préférence d'après les courbes de préférence d'habitat, appliquées dans cette étude à la cohorte $0+$ de truite (BOVEE, 1982 ; SOUCHON et al., 1989). La Surface Pondérée Utile (SPU, en $\mathrm{m}^{2}$ ) de chaque cellule est alors obtenue en multipliant la surface mouillée de la cellule par le produit des trois coefficients de préférence (variant de 0 à 1). Les SPU élémentaires sont ensuite additionnées pour obtenir la SPU de la station (SPU totale), et cela pour différents débits. Le résultat de la simulation est une courbe d'évolution de la SPU en fonction du débit SPU(Q).

La prise en compte du temps dans ce résultat a consisté à coupler la courbe SPU(Q) à des chroniques de débit $Q(t)$ pour obtenir des chroniques temporelles (t) de conditions d'habitat potentiel SPU(t). Sur chacun des deux cours d'eau étudiés, une chronique de débits moyens journaliers $Q(t)$ était disponible, de 1983 à 1993. A titre de comparaison, une chronique de débit a été enregistrée au pas de temps d'une minute, pendant une durée de 100 jours, sur une station soumise à des éclusées, située sur l'Ance du Nord en Haute-Loire (VALENTIN et al., 1994a).

L'analyse en Durées Continues d'Habitat Limitant (DCHL) permet de recenser les durées continues pendant lesquelles la SPU de la station est inférieure à un seuil donné, exprimé en pourcentage de la SPU maximum lue sur la courbe SPU(Q). Ces durées continues sont classées de la plus longue à la plus courte. Elles sont représentées graphiquement en utilisant comme ordonnée la durée en nombre de jours et comme 
abscisse le pourcentage que représente ce nombre de jours par rapport à la durée totale d'étude (Fig. 1). Ces pourcentages sont cumulés sur l'axe des abscisses pour obtenir, en reliant les points, une courbe permettant une bonne visualisation du classement des durées continues. Plusieurs courbes DCHL, correspondant à différents seuils de SPU, peuvent être représentées sur le même graphe.

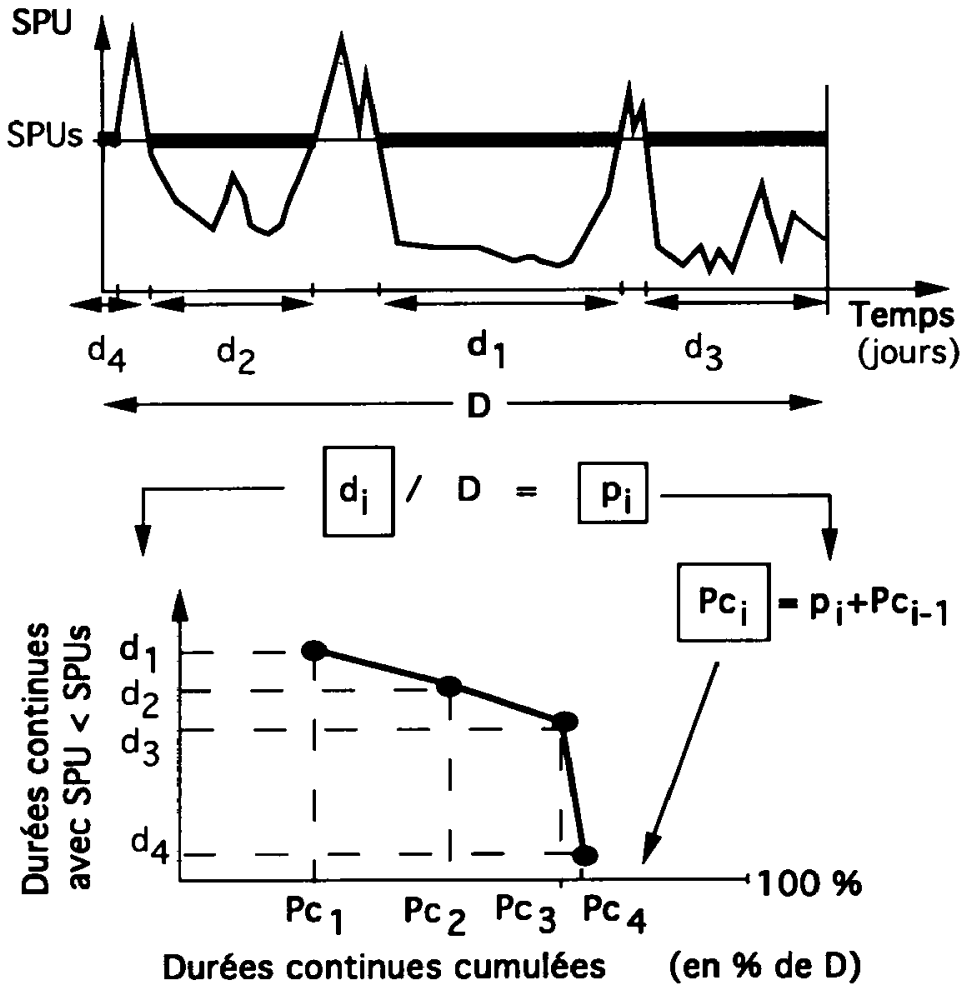

Figure 1 : Principe de construction des courbes DCHL à partir d'une chronique de SPU $(t)$. SPUs représente un seuil d'étude de la chronique. $D$ est le nombre total de jours étudiés. $d_{i}(i=1,4)$ sont des durées continues de SPU <SPUs, classées de la plus longue $\left(d_{1}\right)$ à la plus courte $\left(d_{4}\right) . p_{i}(i=1,4)$ est le pourcentage de chaque di par rapport à $\mathrm{D}$. $\mathrm{Pc}_{\mathrm{j}}$ est le pourcentage cumulé de chaque di.

Figure 1 : Principle of the elaboration of Continuous Under Threshold Habitat Duration Curves (CUT curves). SPUs represents a threshold to study the chronicle. $D$ is the total number of days of the studied period. $d_{i}(i=1.4)$ are continuous durations during which Weighted Usable Area (SPU in French) is lower than SPUs. They are ranked from the longest $\left(d_{1}\right)$ to the shortest $\left(d_{4}\right) \cdot p_{i}(i=1.4)$ is the percentage of total $D$ corresponding to each di. $P c_{i}$ is the cumulative percentage for each $d i$.

\section{Etude des populations}

La structure des populations de truite des deux stations naturelles a été examinée lors de neuf campagnes de pêche au total (Tableau I). Les pêches électriques ont été réalisées en deux passages successifs, avec estimation finale des effectifs par la méthode de CARLE et STRUB (1978), préconisée par GERDEAUX (1987). 
Tableau I : Dates des campagnes de pêche électrique réalisées sur les deux cours
d'eau naturels, la Semine et la Boralde de St Chély.

Table I : Dates of electrofishing performed in both natural streams, the Semine and the Boralde of St Chély.

\begin{tabular}{ccc}
\hline & \multicolumn{2}{c}{ Rivières } \\
\hline$n^{\circ}$ \\
campagne & Semine & B. St Chély \\
\hline 1 & & \\
2 & $09-85$ & $10-86$ \\
3 & $07-91$ & $09-87$ \\
4 & $08-92$ & $10-91$ \\
5 & $07-93$ & $10-92$ \\
\hline
\end{tabular}

Dans les deux stations naturelles, des courbes DCHL pour le stade $0+$ ont été construites (avec des seuils empiriquement choisis égaux à $50 \%$ et $75 \%$ de la SPU max) entre l'émergence (début avril) et la date de chaque pêche. Pour chacun de ces deux seuils, nous avons retenu le pourcentage de la plus longue durée continue ainsi que le pourcentage cumulé total des durées continues sur la durée totale étudiée. Pour chaque seuil, nous avons alors testé les relations entre les effectifs de $0+$ et chacun de ces pourcentages.

\section{RÉSULTATS}

Dans les deux cours d'eau naturels (Semine et Boralde de St Chély), l'allure des courbes DCHL peut être différente selon les années. A titre d'exemple (Fig. 2), sur la Boralde de St Chély, le seuil de SPU à $75 \%$ de la SPU maximum est, en 1986, non dépassé pendant 48 jours consécutifs, soit $23 \%$ de la durée totale d'enregistrement (point indiqué par la flèche sur la figure 2). En 1991, ce seuil est continûment non dépassé pendant de petites durées (inférieures à 5 jours), et pendant relativement peu de temps au total (moins de $8 \%$ de la durée totale). L'année 1992 est intermédiaire, avec de nombreuses durées continues moyennes (inférieures à 20 jours) de non-dépassement du seuil pendant un temps total assez élevé (plus de $35 \%$ du temps).

Les résultats des campagnes de pêche réalisées sur les deux cours d'eau naturels ont montré une fluctuation des effectifs de la cohorte 0+ d'une campagne à l'autre. L'étude des conditions d'habitat des $0+$ entre l'émergence et la date de pêche sur les deux cours d'eau montre une diminution du nombre de $0+$ lorsque le pourcentage de la plus longue durée continue de non-dépassement du seuil $75 \%$ de la SPU maximum pour les $0+$ augmente (régression exponentielle, $\left.y=263.6^{\star} 10^{(-0.072 x)}, r^{2}=0,69, p=0,006\right)$. Les tests effectués entre les effectifs de $0+$ et chacun des trois autres types de pourcentages retenus (pourcentage de la plus longue durée continue de non-dépassement du seuil $50 \%$, pourcentage total des durées continues de non-dépassement du seuil $75 \%$ et pourcentage total des durées continues de non-dépassement du seuil $50 \%$ ) n'étaient pas significatifs.

En situation d'éclusées (Ance du Nord) les variations de débit très fréquentes entraînent de très fortes variations de SPU pour la cohorte 0+ (Fig. 3a). Les courbes DCHL (Fig. 3b) reflètent la rapidité de fluctuation des SPU car les durées continûment non dépassées sont très courtes. Les éclusées étant fréquentes, ces courtes durées continues arrivent souvent et représentent un pourcentage du temps élevé (jusqu'à $40 \%$ ). L'étendue des ordonnées des courbes DCHL obtenues est donc faible et concerne des durées nettement inférieures à celle obtenues dans le cas d'un régime naturel. De plus, du fait de variations fréquentes entre la SPU maximum et le seuil à $25 \%$, les courbes représentées pour les seuils à 90,70 et $50 \%$ (à titre d'exemple) sont très proches (Fig. $3 \mathrm{~b}$ ). 


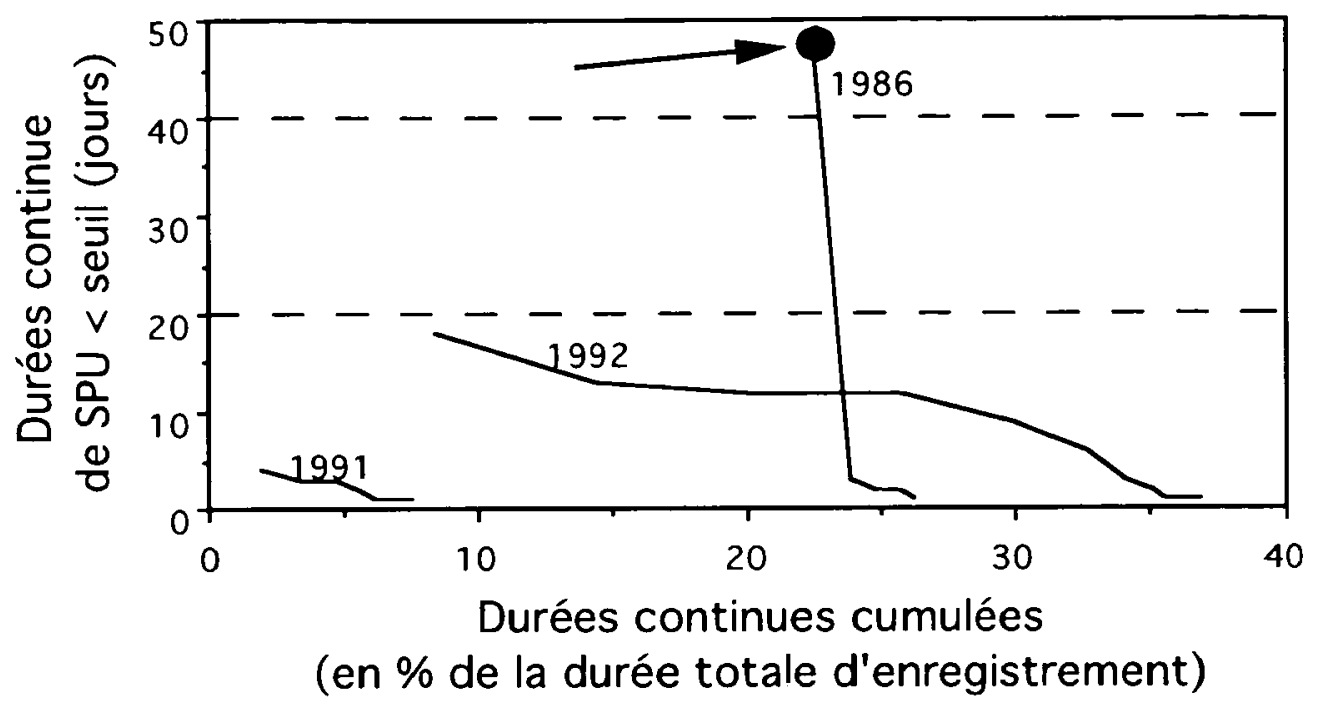

Figure 2 : Courbes DCHL pour le stade $0+$ au seuil de $75 \%$ de la SPU maximum, sur la période de l'émergence à la date de pêche, pour trois années d'enregistrement $(1986,1991$ et 1992), sur la Boralde de Saint-Chély. La flèche indique le point pris en exemple dans le texte.

Figure 2 : CUT curves for YOY corresponding to the threshold $75 \%$ of maximum SPU, from emergence to the sampling date, over three years of records (1986, 1991 and 1992), on the Boralde of Saint-Chély River. The arrow represents the example mentioned in the text.

\section{DISCUSSION}

L'étude de l'ensemble des courbes DCHL obtenues en milieu naturel permettent de confirmer les premiers résultats concernant cette méthodologie obtenus sur le stade reproduction par CAPRA et al. (1994). Les résultats présentés ici ont montré que lorsque les durées continues de non-dépassement de seuils de SPU sont grandes, les populations de truite sont défavorisées. La partie supérieure droite du plan DCHL (Fig. 4) correspond donc à une situation naturelle défavorable (longue durée continue de SPU faible), expliquant d'éventuelles anomalies dans la structure des populations. II existe un gradient de types de courbes, de celles qui représentent une ou plusieurs longues durées (1986 et 1992, Fig. 2) à celles représentant des petites durées peu nombreuses (ex. 1991 Fig. 2). On peut ainsi distinguer une zone d'habitat non limitant et une zone d'habitat défavorable en milieu naturel (Fig. 4). Cependant le domaine de validité de ces conclusions concerne les cours d'eau de type "naturel», en dehors de périodes exceptionnelles (crues ou étiages très sévères), en supposant de plus que l'habitat constitue le facteur principal agissant sur la répartition spatiale des individus.

La lecture des chroniques de SPU est très difficile en situation de variabilité extrême, comme le suggère l'exemple présenté à la figure $3 \mathrm{a}$. Des outils supplémentaires sont donc indispensables pour aider à quantifier les variations d'habitat consécutives aux éclusées. L'interprétation de la zone occupée par des courbes DCHL construites en situation d'éclusées est différente de la situation naturelle et pose de nouvelles questions. Quelles sont les conséquences de courtes durées continues de SPU faibles se produisant souvent ? Cette situation est-elle plus ou moins pénalisante qu'une seule longue durée de faible SPU ? Quel est le facteur limitant, sachant que des seuils très différents donnent le même type de courbe 

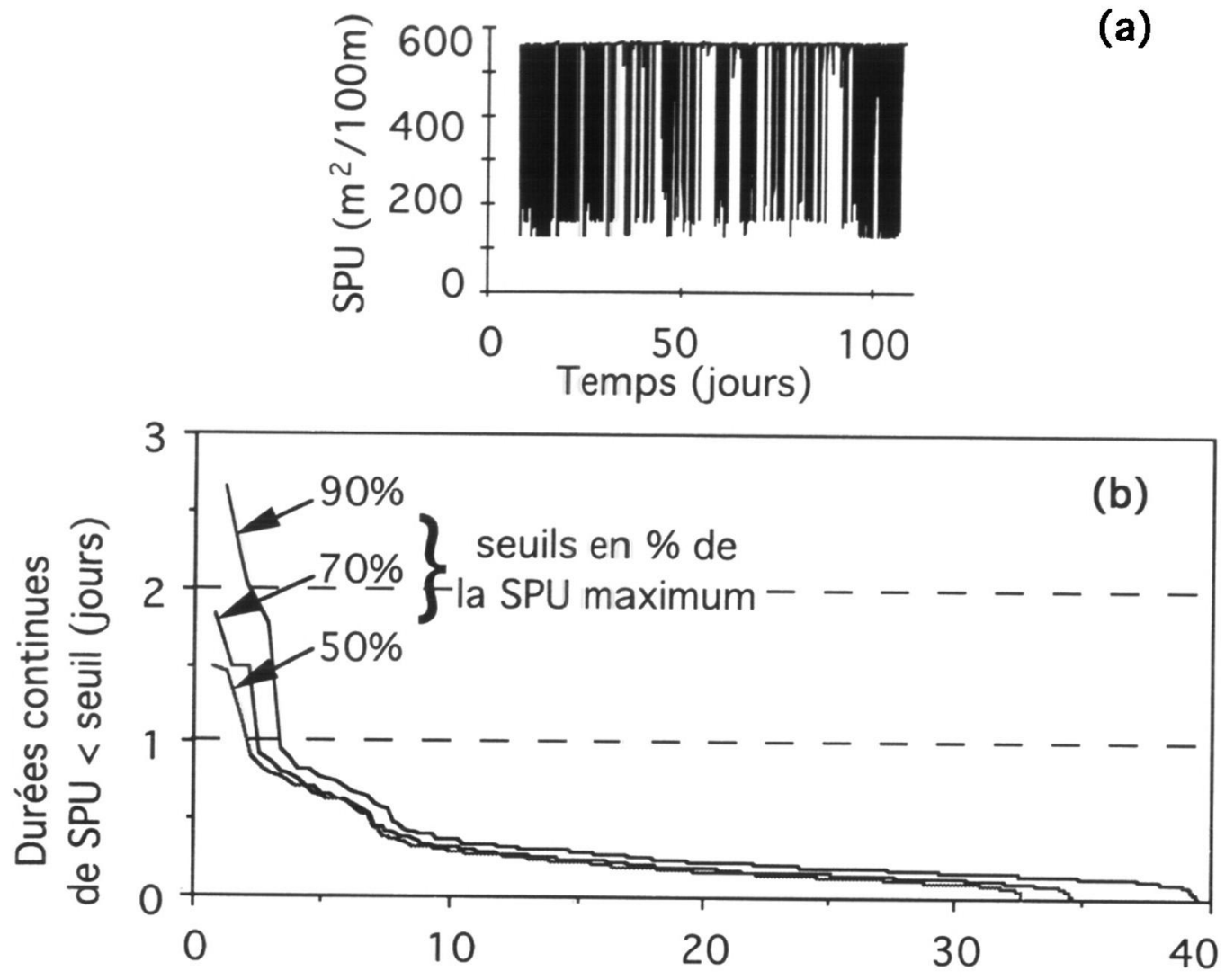

Figure 3 : Chroniques d'habitat en situation d'éclusées. (a) Chronique de SPU pour truite fario 0+ sur l'Ance du Nord, obtenue à partir d'enregistrements du débit en pas de temps variable, pendant 100 jours en 1993. (b) Courbes DCHL pour le stade $0+$, sur la chronique (a), en fonction de trois seuils différents.

Figure 3 : Habitat time series in regulated conditions. (a) WUA chronicle for YOY brown trout in the Ance du Nord River, from records at variable time steps, over 100 days in 1993. (b) CUT curves for YOY corresponding to chronicle (a), for three different thresholds.

en situation d'éclusées? A partir de quelle fréquence la variabilité rapide des conditions d'habitat devient-elle préjudiciable ? On ne connaît pas encore précisément les mécanismes limitants dans des conditions d'habitat aussi variables.

L'interprétation des variations de SPU et notamment à fort débits en régime fluctuant ne peut s'entendre qu'en tenant compte du rapport disponibilité/utilisation des refuges. Ceux-ci restent malheureusement difficiles à quantifier et à estimer à partir des modèles hydrauliques classiques. Ils ne sont donc pas bien pris en compte dans le calcul des SPU du fait que les courbes de préférence ont été construites en situation non perturbée. NIEMELA (1989) a montré que les préférences de truite arc-en-ciel adultes (Oncorhynchus mykiss) changeaient pendant des éclusées. Cependant, la construction de nouvelles courbes de préférence applicables à fort débit ne nous semble pas pertinente car on aurait 


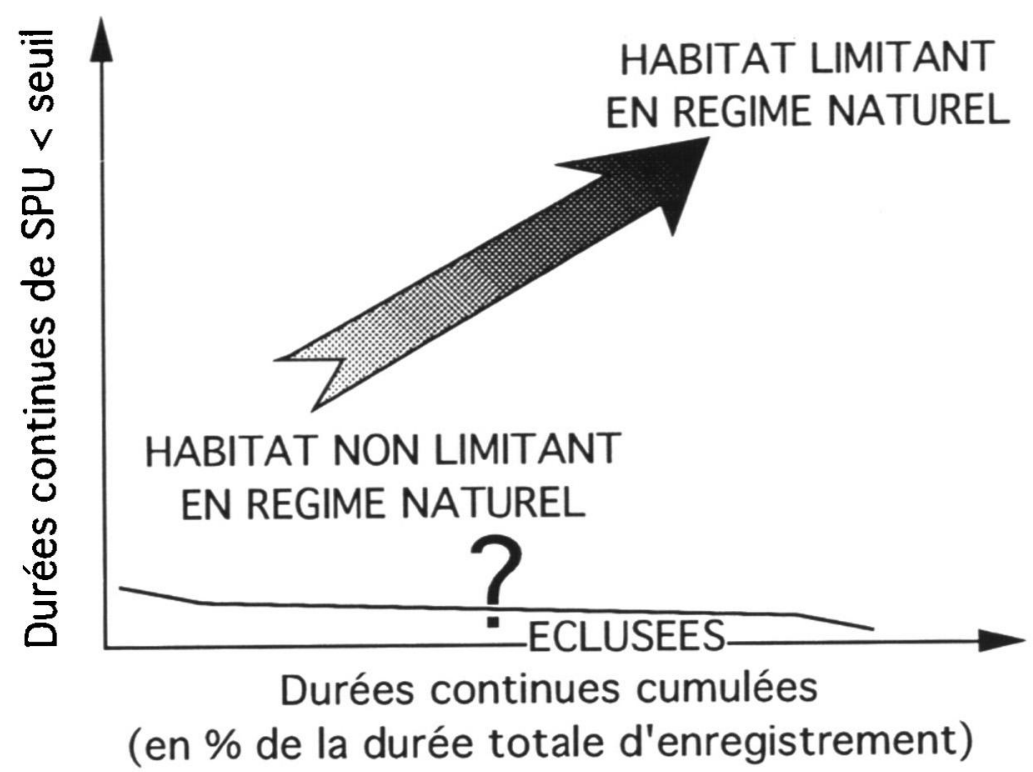

Figure 4 : Représentation schématique des zones d'emplacement des courbes DCHL en régime naturel et en situation d'éclusées.

Figure 4 : Schematic representation of CUT curve location in natural and regulated flow regimes.

alors tendance à adapter le modèle à des résultats obtenus dans un cas particulier. En revanche, la compréhension des mécanismes expliquant la répartition des poissons à débit d'éclusées devrait s'appuyer sur la recherche d'autres facteurs que la SPU pour quantifier la capacité d'accueil tels que les refuges disponibles (GORE et al., 1994) ou les aptitudes individuelles à trouver des refuges (VALENTIN et al., 1994b) ou à résister au courant.

\section{REMERCIEMENTS}

Les campagnes de terrain ont été effectuées avec l'aide de P. ROGER, M. PHILIPPE, M. POUILLY, L. MARIDET, R. BONNARD, Y. SOUCHON et J.G. WASSON (Laboratoire d'Hydroécologie Quantitative, CEMAGREF, Lyon, France). Un support financier a été apporté par le CEMAGREF et le "Programme Interdisciplinaire de Recherche en Environnement: Habitat-Poissons". L'étude sur les éclusées hydroélectriques a été en partie financée par EDF (contrat $n^{\circ} 2 \mathrm{~K} 1867$ ). Que toutes ces aides précieuses soient vivement remerciées.

\section{BIBLIOGRAPHIE}

BOVEE K.D., 1982. A guide to stream habitat analysis using the Instream Flow Incremental Methodology. Instream Flow Information Paper $n^{\circ} 12$, Western Energy and Land Use Team, U.S. Fish and Wildlife Service, Fort Collins, Colorado, FWS/OBS 82/86, 248 p.

CAPRA H., BREIL P., SOUCHON Y., 1995. A new tool to interpret magnitude and duration of fish habitat variations. Actes du Colloque SISORS II Czech Republic, 3-6 Août 1994. Regul. Riv. : Res. \& Manage., 10, 281-289. 
CARLE F.L., STRUB M.R., 1978. A new method for estimating population size from removal data. Biometrics, 34, 621-630.

GERDEAUX D., 1987. Revue des méthodes d'estimation de l'effectif d'une population par pêches successives avec retrait, programme d'estimation d'effectif par la méthode de Carle et Strub. Bull. Fr. Pêche. Piscic., 304, 13-21.

GINOT V., SOUCHON Y., 1992. "EVHA». Un système informatique pour évaluer l'habitat du poisson. In ADEME, Insertion des petites centrales hydroélectriques dans l'environnement, Sophia Antipolis, décembre 1992, 157-166.

GORE J.A., NIEMELA S., RESH V., STATZNER B., 1994. Near-substrate hydraulic conditions under artificial floods from peaking hydropower operation : a preliminary analysis of disturbance intensity and duration. Regul. Riv. : Res. \& Manage., 9, 15-34.

JOWETT I.G., 1992. Models of the abundance of large brown trout in New Zealand rivers. N. Am. Fish. Manag., 12, 417-432.

MILHOUS R.T., UPDIKE M.A., SCHNEIDER D.M., 1989. Physical habitat simulation system reference manual - version II. Instream Flow Information Paper $n^{\circ} 26$, Fish and Wildlife Service, Office of Biological Service, Ft Collins, FWS/OBS 89/16.

NIEMELA S.L., 1989. The influence of peaking hydroelectric discharges on habitat selection and movement patterns of Rainbow trout (Oncorhynchus mykiss). MSc thesis, Tennessee Technological University, Cookeville, $118 \mathrm{p}$.

OBERLIN G., BEN HANSOUR H., ORTIZ R., 1989. Generalization and Standardization for 3 types of flow-duration-frequency curves in flood regions. Description and transfer. Friends in Hydrology, UNESCO, IASH, Bolkesjö, Norway (disponible au CEMAGREF, division Hydrologie-Hydraulique).

ORTH D.J., 1987. Ecological considerations in the development and application of instream flow habitat models. Regul. Riv. : Res. \& Manage., 1, 171-181.

POUILLY M., VALENTIN S., CAPRA H., GINOT V., SOUCHON Y., 1995. Méthode des microhabitats : principes et protocoles d'application. Bull. Fr. Pêche. Piscic., 336, 41-54.

REISER D.W., WESCHE T.A., ESTES C., 1989. Status of instream flow legislation and practices in North America. Fisheries, 14 (2), 22-29.

SCOTT D., SHIRVELL C.S., 1987. A critique of the instream flow incremental methodology and observations on flow determination in New Zealand. In Craig J.F. and Kemper J.B., Regulated streams: Advances in ecology, 27-44, Plenum Press, New York.

SHULER S.W., NEHRING R.B., 1994. Using the Physical Habitat Simulation model to evaluate a stream habitat enhancement project. Rivers, 4 (3), 175-193.

SOUCHON Y., TROCHERIE F., FRAGNOUD E., LACOMBE C., 1989. Les modèles numériques des microhabitats des poissons : application et nouveaux développements. Rev. Scie. Eau, 2, 807-830.

VALENTIN S., LAUTERS F., SABATON C., BREIL P., SOUCHON Y., 1994a. Temporal variations of physical habitat for brown trout (Salmo trutta fario, L. 1758) in hydropeaking situations : methodological approach tested in two French streams. Regul. Riv. : Res. \& Manage., Actes du Colloque "First International Symposium on Habitat Hydraulics", Trondheim, Norway, 18-20 Août 1994, 277-293.

VALENTIN S., SEMPESKI P., SOUCHON Y., GAUDIN P., 1994b. Short-term habitat use by young grayling. Thymallus thymallus $L$., under variable flow conditions in an experimental stream. Fisheries Management and Ecology, 1, 57-65. 